\title{
ANÁLISE DA EVOLUÇÃO DAS EMPRESAS SUPERMERCADISTAS BRASILEIRAS NO PERÍODO DE 2002 A 2006
}

\author{
Analysis of the development of the brazilian \\ supermarkets between 2002 to 2006
}

Envio 04.08.08 / Aceite 15.10.08

\author{
Marcos Roberto Luppe ${ }^{1}$ \\ Claudio Felisoni de Angelo ${ }^{2}$ \\ Flávia Angeli Ghisi ${ }^{3}$ \\ Patrícia de Salles Vance ${ }^{4}$
}

\begin{abstract}
Resumo
A partir da década de 1990, a estabilidade econômica, aliada ao avanço tecnológico, à globalização e ao surgimento de novos formatos, alterou significativamente a forma de o varejo supermercadista operar no Brasil, exigindo o aprimoramento da gestão operacional dessas empresas. As transformações descritas estimularam a concentração do mercado, porém o modo como as empresas estão agrupadas em função de determinados indicadores ainda desperta dúvida. O presente trabalho implementa técnicas de ANACOR e HOMALS em grupos supermercadistas brasileiros, com o intuito de analisar o impacto de três indicadores no comportamento dessas empresas. Para o desenvolvimento deste trabalho, utilizaram-se os dados secundários provenientes do ranking das maiores empresas supermercadistas atuantes no Brasil entre 2002 e 2006. Foram coletados dados referentes à região em que a sede da
\end{abstract}

\footnotetext{
1Doutorando do Departamento de Administração da Faculdade de Economia, Administração e Contabilidade da Universidade de São Paulo - FEA/USP. Av. Prof. Luciano Gualberto, 908 - Cidade Universitária. Prédio FEA 1 - sala G-121. CEP: 05508-900 - São Paulo - SP. Email: mluppe@usp.brmluppe@usp.br.

${ }^{2}$ Professor Titular do Departamento de Administração da Faculdade de Economia, Administração e Contabilidade da Universidade de São Paulo - FEA/USP. Av. Prof. Luciano Gualberto, 908 - Cidade Universitária. Prédio FEA 1 - sala G-121. CEP: 05508-900 - São Paulo - SP. E-mail: cfa@usp.brcfa@usp.br.

${ }^{3}$ Professora Doutora do Mestrado Profissional da Fundação Instituto de Administração - FIA. Rua Navarro de Andrade, 152, sala T3. CEP: 05418-020 - São Paulo- SP. E-mail: flaviag@provar.orgflaviag@provar.org.

${ }^{4}$ Doutoranda do Departamento de Administração da Faculdade de Economia, Administração e Contabilidade da Universidade de São Paulo - FEA/USP. Av. Prof. Luciano Gualberto, 908 - Cidade Universitária. Prédio FEA 1 - sala G-121. CEP: 05508-900 - São Paulo - SP. E-mail: patricia_vance@provar.orgpatricia_vance@provar.org.
} 
empresa está instalada, faturamento por check-out, faturamento por $\mathrm{m}^{2}$ e faturamento por funcionário, dos anos de 2002 a 2006.

Palavras-chave: Supermercados; Técnica de ANACOR; Técnica de HOMALS.

\begin{abstract}
From the 90's, the economic stability, allied with the technological advance, the globalization and the emerging of new formats, modified significantly the way that the supermarket sector operates in Brazil, demanding the improvement of the operational management of these companies. These changes stimulated the market concentration, however the way the companies are connected, according to certain indicators, is still doubtful. This paper implements ANACOR and HOMALS statistic techniques in Brazilian supermarket chains, aiming at analyzing the impact of three specific indicators in the development of these companies. In order to develop this research, secondary data from the ranking of the biggest supermarket chains in Brazil between 2002 and 2006 were used. The data collected refers to the regions where the headquarters of the companies are placed, by check-out, by yd2 and by employee invoices, from 2002 to 2006.
\end{abstract}

Keywords: Supermarkets; ANACOR; HOMALS.

\title{
1 Introdução
}

Na economia brasileira atual, de forma semelhante ao que acontece em alguns países desenvolvidos, o setor terciário e de serviços apresenta-se como o de maior importância. Entre os vários serviços ofertados por esse setor, destaca-se o comércio e, mais especificamente, o varejo, uma atividade cuja relevância não decorre apenas de sua enorme expressão econômica, mas também por ser o elo final da cadeia de abastecimento. Ademais, por manter um contato direto com os consumidores, serve como um indicador das tendências do mercado de consumo e da distribuição de bens em geral.

Durante muitas décadas, os aspectos operacionais relacionados à administração varejista foram negligenciados e as vastas e rentáveis possibilidades oferecidas pelo mercado financeiro permitiam que as empresas do setor compensassem com grande facilidade seus problemas operacionais (BLECHER, 2001). Por outro lado, a estabilidade econômica alterou significativamente a forma de atuação de grupos varejistas e reestruturou o mercado de consumo. A nova realidade econômica, aliada ao avanço tecnológico, à globalização e ao surgimento de novos formatos, alterou significativamente a forma de o varejo operar, abrindo espaço para questionamentos e aprimoramento do seu gerenciamento.

As transformações descritas estimularam o aumento de tamanho das empresas supermercadistas (SESSO FILHO, 2001), porém o modo como as empresas estão agrupadas em função de determinados indicadores ainda desperta dúvida. E, dessa forma, o desenvolvimento deste trabalho utiliza os dados do ranking da Revista SuperHiper para analisar o comportamento das redes supermercadistas em cada uma das regiões brasileiras nos últimos cinco anos (20022006) em relação a alguns indicadores de desempenho dessas empresas.

Este ranking é desenvolvido anualmente pela Associação Brasileira de Supermercados (ABRAS), em parceria com a Nielsen. Nesse ranking, são apresentados dados referentes as 500 maiores empresas supermercadistas. Essas empresas são classificadas pelo faturamento bruto anual e, além desse indicador, são apresentados os dados do número de check-outs, da 
área de vendas em $\mathrm{m}^{2}$, do número de lojas e do número de funcionários para cada uma das empresas.

Assim, com o objetivo de analisar o impacto de três indicadores de desempenho (faturamento por check-out, faturamento por $\mathrm{m}^{2}$ e faturamento por funcionário) no comportamento dessas empresas em cada uma das regiões brasileiras, utilizam-se os métodos multivariados de análise de correspondências (ANACOR) e análise de homogeneidade (HOMALS). O tratamento dos dados se deu por meio do software estatístico SPSS (Statistical Package for Social Sciences).

Por meio da aplicação inicial da ANACOR para cada um dos anos do estudo, são verificadas se existem associações entre cada um dos indicadores de desempenho e as respectivas regiões brasileiras e, com a definição destas, aplica-se a HOMALS para a identificação das associações entre todas as variáveis observacionais analisadas, além da sua visualização em um espaço dimensional.

Embora essa reflexão possa, provavelmente, aplicar-se a todos os segmentos que compõem os setores produtivos e de prestadores de serviços no Brasil, não há dúvidas que o ramo composto pelos supermercados mereça atenção especial (ANGELO, SILVEIRA e SIQUEIRA, 2001). Atualmente, grande parte da comercialização de bens no Brasil se realiza por meio desse tipo de comércio, e os supermercados vêm expandindo os seus ramos de atuação e ampliando o sortimento dos produtos oferecidos. As suas atividades, outrora restritas à comercialização de produtos alimentares e de higiene e limpeza, estão sendo expandidas para outros segmentos, como os relacionados a bens duráveis, semiduráveis e têxteis, entre outros.

Este trabalho está organizado da seguinte forma: a próxima seção descreve a evolução e o panorama do setor supermercadista no Brasil. Na seqüência, aborda-se o método de pesquisa e uma revisão teórica dos conceitos. Em seguida, os resultados são apresentados e analisados e, por fim, são apresentadas as conclusões e as possíveis extensões do presente estudo.

\section{Evolução e panorama do setor supermercadista no Brasil}

Com a política de redução drástica do ritmo inflacionário, introduzida em 1994 com o Plano Real, os supermercados estão sendo expostos a desafios cada vez maiores, no sentido de atingirem a excelência em operações e produtividade (MARQUES e NORONHA, 2001). Antes de 1994, as empresas compensavam seus problemas operacionais com os ganhos obtidos por meio da aplicação financeira, que, quando acompanhava a inflação, podia atingir patamares de até $80 \%$ ao mês. Com a estabilidade dos patamares inflacionários, os grupos supermercadistas deixaram de lucrar com as aplicações financeiras. A necessidade de ofertar produtos e serviços de melhor qualidade, diferenciados e a preços coerentes e competitivos, obrigou os supermercadistas a se preocuparem com as operações e com a oferta. A satisfação do consumidor passa a ser extremamente importante para o bom desempenho dos grupos supermercadistas (MARQUES e NORONHA, 2001).

Em praticamente cinco décadas de implantação no Brasil, o supermercado tornou-se um elemento indissociável do modo de vida e das preocupações de qualquer consumidor. Os principais supermercados foram instalados no país a partir da década de 1960 e, na década seguinte, houve sedimentação das grandes cadeias, inclusive com a abertura dos primeiros hipermercados (MARQUES e NORONHA, 2001).

A década de 1980 foi caracterizada por instabilidades políticas e econômicas no País, enquanto a década de 1990 foi marcada pela estabilidade econômica e o fim da ciranda financeira, exigindo fortes ajustes por parte do varejo, caracterizada, então, por uma enorme 
competição e por forte concentração no setor supermercadista, inclusive com o aparecimento de grandes aquisições e expansões realizadas por alguns grupos supermercadistas (ROJO, 1998). $\mathrm{Na}$ transição dos anos 1990 para o novo século, esse processo foi muito intenso, tanto pela incorporação de várias redes nacionais pelas estrangeiras, como também pela aquisição das redes de pequeno e médio portes pelas redes nacionais maiores, resultando em uma mudança estrutural do mercado, com a presença de maiores concentrações nessa área de varejo (GHISI, CAMARGO e MARTINELLI, 2003). Como empresas varejistas estrangeiras, citam-se: o WallMart (norte-americano), o Carrefour (francês) e o Casino (francês). O ano de 2005 foi de acomodação das aquisições antes realizadas pelas empresas do setor (SUPERHIPER, 2006).

Em 1994, mesmo ano da introdução do Plano Real, o WallMart chega ao Brasil. Dez anos depois, adquire a rede Bompreço, do grupo holandês Royal Ahold, e expande suas operações na Região Nordeste. Com isso, assume a terceira posição no ranking da ABRAS. Em 2005, adquire as operações supermercadistas do grupo português Sonae no Brasil, com forte atuação na Região Sul (WALLMART, 2008).

Ocorrem também, nesta década, várias aquisições por parte do Grupo Pão de Açúcar e do Carrefour. Esta última empresa, de origem francesa, adquire redes regionais, como Planaltão, Roncetti, Mineirão, Rainha, Dallas e Continente, e lança o formato Carrefour Bairro (CARREFOUR, 2008). O Grupo Pão de Açúcar adquire, entre outras redes: Supermercados $A B C(R J)$, Sé Supermercados (SP) e associa-se à rede Sendas (RJ). A partir de 2005, o controle acionário deste Grupo é compartilhado igualmente pelo empresário Abílio Diniz e o Grupo Casino, também de origem francesa (GRUPO PÃO DE AÇÚCAR, 2008).

O Quadro 1 traz um resumo pontual de mais alguns relevantes acontecimentos no setor supermercadista. 


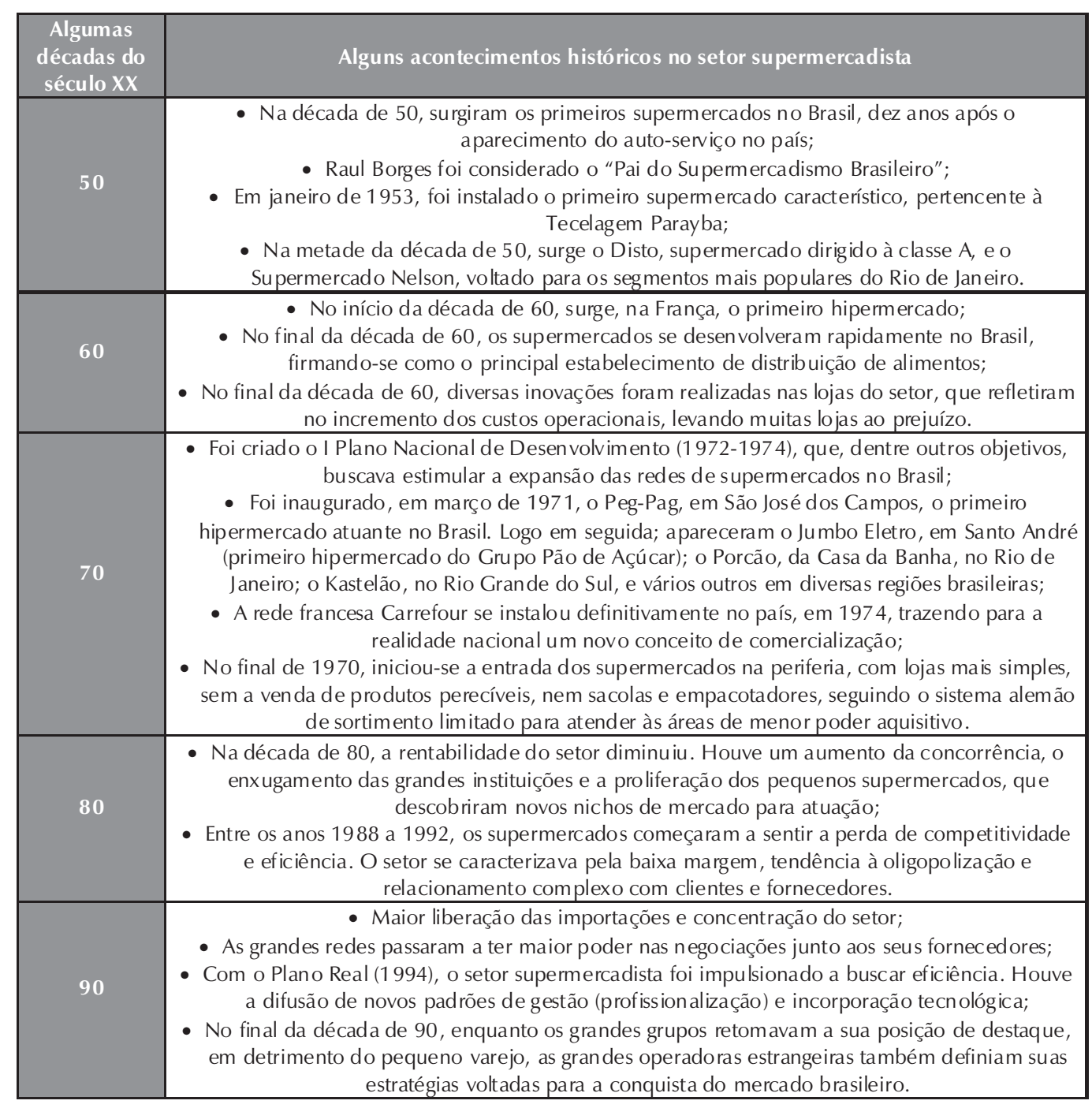

Fonte: Cyrillo (1987); Fox (1992); Garry (1992); O’Neill (1992); Garry (1994); Connor e Schiek (1997); John (2000); Quental, Silva e Leite (2001); Sesso Filho (2003) e Dieese (2003).

Quadro 1 - Retrospectiva histórica de relevantes acontecimentos no setor supermercadista.

De acordo com a Revista SuperHiper (2006), os dez maiores grupos supermercadistas presentes no Brasil apresentam um faturamento bruto de 51,4 bilhões de reais, o que equivale, em valores, a 2,2\% do PIB nacional. A seguir, na Tabela 1, é apresentada a evolução do faturamento desses grupos nos últimos cinco anos. 
Tabela 1 - Evolução do faturamento bruto $(\mathrm{R} \$$ ) dos dez maiores grupos supermercadistas brasileiros no período de 2002-2006

\begin{tabular}{l|l}
\hline ANO & $\begin{array}{c}\text { FATURAMENTO BRUTO DOS 10 MAIORES } \\
\text { GRUPOS SUPERMERCADISTAS (R\$) }\end{array}$ \\
\hline 2002 & $35.919 .218 .388,00$ \\
\hline 2003 & $39.062 .250 .782,00$ \\
\hline 2004 & $44.409 .561 .740,00$ \\
\hline 2005 & $48.292 .221 .206,00$ \\
\hline 2006 & $51.375 .418 .109,00$ \\
\hline
\end{tabular}

O Quadro 2 apresenta o ranking dos 10 maiores supermercadistas brasileiros entre os anos de 2002 e 2006, em ordem decrescente de faturamento, de acordo com a Revista SuperHiper.

\begin{tabular}{|c|c|c|c|c|c|}
\hline & 2002 & 2003 & 2004 & 2005 & 2006 \\
\hline 1 & $\begin{array}{l}\text { COMPANHIA BRASILEIRA DE } \\
\text { DISTRIBUIÇÃO }\end{array}$ & $\begin{array}{l}\text { COMPANHIA BRASILEIRA DE } \\
\text { DISTRIBUIÇÃO }\end{array}$ & $\begin{array}{l}\text { COMPANHIA BRASILEIRA DE } \\
\text { DISTRIBUIÇÃO }\end{array}$ & $\begin{array}{l}\text { COMPANHIA BRASILEIRA DE } \\
\text { DISTRIBUIÇ̃̃O }\end{array}$ & $\begin{array}{l}\text { COMPANHIA BRASILEIRA DE } \\
\text { DISTRIBUIČÃOO }\end{array}$ \\
\hline 2 & CARREFOUR & CARREFOUR & CARREFOUR & CARREFOUR & WAL-MART BRASIL \\
\hline 3 & BOMPREÇO & SONAE & WAL-MART BRASIL & WAL-MART BRASIL LTDA & CARREFOUR \\
\hline 4 & SONAE & BOM PREÇO & SONAE & CIA ZAFFARI & GBARBOSA COMERCIAL \\
\hline 5 & SENDAS & SENDAS SA & ZAFFARI & GBARBOSA COMERCIAL LTDA & ZAFFARI \\
\hline 6 & WAL-MART BRASIL & WAL-MART BRASIL & DMA DISTRIBUIDORA & DMA DISTRIBUIDORA & DMA DISTRIBUIDORA \\
\hline 7 & CIA. ZAFFARI & CIA ZAFFARI & GBARBOSA COMERCIAL & IRMÃOS BRETAS & IRMÃOS BRETAS \\
\hline 8 & C. BARBOSA COMERCIAL & COOP & IRMÃOS BRETAS & COOP & PREZUNIC \\
\hline 9 & COOP & GBARBOSA COMERCIAL & COOP & ANGELONI & ANGELONI \\
\hline 10 & IRMÃOS BRETAS & IRMAOS BRETAS & ANGELONI & PREZUNIC & COOP \\
\hline
\end{tabular}

Quadro 2 - Evolução temporal dos 10 maiores grupos supermercadistas no Brasil entre 2002 e 2006.

Desse modo, após a apresentação dos principais conceitos que permeiam este trabalho, no tópico seguinte, será descrito o método de pesquisa desenvolvido e uma revisão teórica das duas técnicas estatísticas multivariadas utilizadas neste estudo.

\section{Método empregado no estudo e revisão teórica dos conceitos}

O presente trabalho implementa técnicas de ANACOR e HOMALS em grupos supermercadistas brasileiros, com o intuito de analisar o impacto de três indicadores no comportamento dessas empresas, sendo que estes indicadores são amplamente utilizados pelo mercado para avaliar o desempenho desse tipo de empresa ao longo do tempo. Para o desenvolvimento deste trabalho, utilizaram-se os dados secundários provenientes do ranking das maiores empresas supermercadistas atuantes no Brasil entre 2002 e 2006 (REVISTA SUPERHIPER, 2003-2007). Foram coletados dados referentes à região em que a sede da 
empresa está instalada, faturamento por check-out, faturamento por m2 e faturamento por funcionário, dos anos de 2002 a 2006. As variáveis coletadas encontram-se no Quadro 3.

\begin{tabular}{|c|c|}
\hline Código & Variável \\
\hline Região & Região da sede da empresa \\
\hline Faturcheck & Faturamento Check Out \\
\hline Faturm2 & Faturamento/m ${ }^{2}$ \\
\hline Faturfunc & Faturamento/Funcionário \\
\hline
\end{tabular}

Fonte: Revista SuperHiper (2003-2007).

Quadro 3 - Variáveis utilizadas no estudo.

A variável região foi codificada segundo as cinco regiões brasileiras, como: $1=$ Sul, $2=$ Sudeste, 3 = Centro-Oeste, $4=$ Nordeste e $5=$ Norte. As variáveis dos indicadores de desempenho foram divididas pelos tercis da distribuição dos valores dessas variáveis, e foram estipulados valores iguais a 1 para o $1^{\circ}$ tercil, iguais a 2 para o $2^{\circ}$ e iguais a 3 para o $3^{\circ}$. Os critérios de divisão são apresentados no Quadro 4.

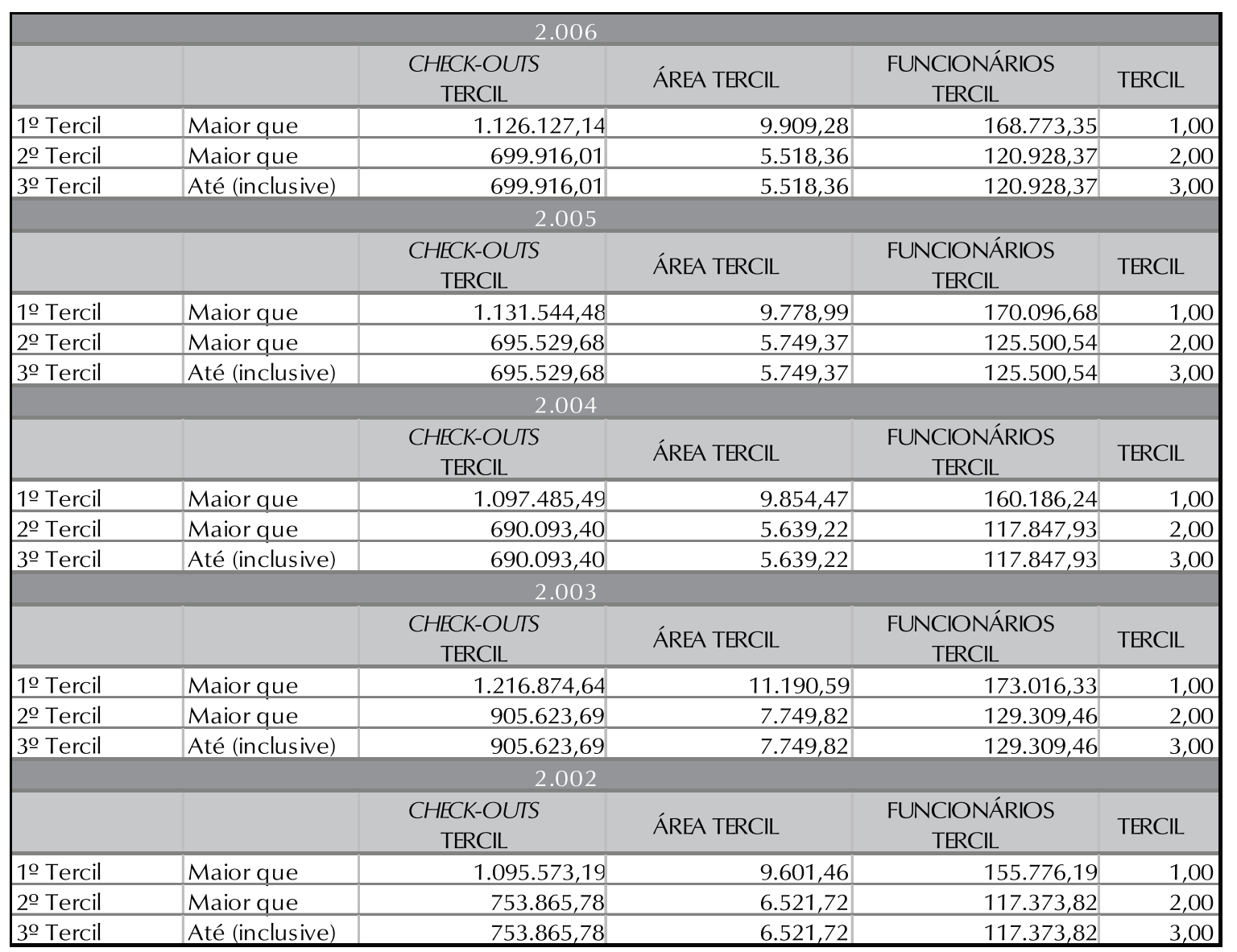

Quadro 4 - Critérios para divisão da amostra das empresas (2002 -2006).

Com base nos dados coletados, foram feitas duas análises. Na primeira, para cada um dos cinco anos analisados, foi verificado se existia associação entre a variável região e cada uma das variáveis de desempenho (faturamento por check-out, faturamento por $\mathrm{m}^{2}$ e faturamento 
por funcionário), e isso foi verificado pela significância dos testes qui-quadrado $\left(\chi^{2}\right)$ nas análises de ANACOR. Na segunda, aplicou-se a HOMALS, com todas as variáveis significativas definidas na etapa anterior, para a análise do impacto dos três indicadores de desempenho, em cada um das regiões brasileiras ao longo dos últimos cinco anos.

\subsection{Análise de correspondência}

Segundo Whitlark e Smith (2001), a análise de correspondência (Anacor) é uma técnica que exibe as associações entre um conjunto de variáveis categóricas nominais em um mapa perceptual, permitindo, dessa maneira, um exame visual de qualquer padrão ou estrutura nos dados. De acordo com Batista, Escuder e Pereira (2004), a análise de correspondência é uma técnica de representação gráfica em projeção plana das relações multidimensionais das distâncias $\chi^{2}$ entre as categorias das variáveis estudadas. Neste trabalho, utilizou-se a projeção simétrica, que permite examinar simultaneamente as relações entre linhas e colunas da tabela de contingência, ou seja, as relações entre todas as categorias de ambas as variáveis. De acordo com Fávero, Belfiore e Fouto (2006), categorias com localização próxima na projeção plana têm relação mais forte do que categorias separadas por distâncias maiores. Qualquer categoria, representada como um ponto no mapa perceptual, pode ser analisada em separado e caracterizada segundo a proximidade das projeções de todas as outras categorias sobre uma reta que ligue seu ponto característico à origem dos eixos do plano de projeção. Quando categorias de uma mesma variável encontram-se em posições próximas no mapa da análise de correspondência, isso sugere que, independentemente de seus conteúdos semânticos, elas podem ser consideradas iguais no que tange à distribuição de massas do total das observações realizadas.

Essa técnica originou-se do analista francês Jean-Paul Benzécri, no início dos anos 1960, e representa uma aplicação de análise multivariada para a exibição de linhas e colunas de uma matriz de dados (principalmente uma tabela de contingência de duas dimensões) como pontos em um espaço dimensional de caráter qualitativo (GREENACRE, 1984). Muitos autores fizeram contribuições significativas em relação à aplicação de modelos multivariados a dados qualitativos, merecendo destaque Haberman (1973), Young (1981), Ludovic, Morineau e Warwick (1984), Caroll, Green e Schaffer (1986), Hoffman e Franke (1986), Benzécri (1992), Nishisato (1993), Greenacre e Blasius (1994), Sharma (1996), Olariaga e Hernández (2000) e Carvalho (2004).

Conforme descrevem Hair et al. (2005), a análise de correspondência é uma técnica multivariada que tem se tornado crescentemente popular para a redução dimensional e o mapeamento perceptual. Entende-se por mapa perceptual a representação visual das percepções de objetos de um indivíduo em duas ou mais dimensões e, normalmente, este mapa tem níveis opostos de dimensões nos extremos dos eixos x e y. Segundo esses autores, a Anacor é uma das técnicas recentemente desenvolvidas para analisar relações não-lineares e dados com respostas categóricas, medidas em termos nominais, e tem como principal objetivo agrupar variáveis altamente associadas, tendo como conseqüência a redução do número de variáveis preditoras do modelo e a representação das relações entre as categorias das variáveis em um mapa perceptual.

A força dessa técnica reside no fato de que ela fornece um meio para examinar as relações não somente entre as variáveis em linha ou em colunas individualmente, mas também entre as variáveis em linha e em coluna conjuntamente, o que implica que, usando essa técnica no contexto deste trabalho, é possível comparar as associações entre a variável região 
e cada uma das variáveis de desempenho (faturamento por check-out, faturamento por $\mathrm{m}^{2} \mathrm{e}$ faturamento por funcionário).

O método consiste de duas etapas básicas, referentes ao cálculo da medida de associação e à criação do mapa perceptual. A Anacor utiliza o teste $\chi^{2}$ para padronizar os valores das freqüências e formar a base para as associações. Com base em uma tabela de contingência, calculam-se as freqüências esperadas e o valor do $\chi^{2}$ para cada célula, considerando-se as diferenças entre as freqüências observadas e as esperadas. Assim, com as medidas padronizadas da associação, a Anacor cria uma medida em distância métrica e cria projeções ortogonais sobre as quais as categorias podem ser alocadas, de forma a representar o grau de associação dado pelas distâncias $\chi^{2}$ em um espaço dimensional.

De acordo com Pestana e Gageiro (2000), recomenda-se inicialmente a realização do teste $\chi^{2}$ para a verificação da existência de dependência entre as duas variáveis e, conseqüentemente, para a avaliação da adequação da aplicação da Anacor. Estes mesmos autores indicam um roteiro básico para a realização da Anacor. Primeiramente, por meio do autovalor (eigenvalue) e das inércias parciais e acumuladas de cada dimensão, julga-se a pertinência de considerá-la para o modelo. Para cada dimensão, o quadrado de cada autovalor é chamado inércia das dimensões e mede a importância de cada dimensão. O quociente entre a inércia de cada dimensão e a inércia total dá a proporção da variância explicada pela dimensão.

O número máximo de dimensões (eixos nos gráficos) que pode ser estimado é um a menos do que o menor número entre a quantia de linhas ou de colunas. Por exemplo, em uma tabela de contingência com três colunas e cinco linhas, o número máximo de dimensões será dois [min (linha, coluna) -1]. Após a determinação da dimensionalidade, os resultados podem ser examinados numa representação gráfica, chamada de mapa perceptual.

A análise desse gráfico faz-se pelo exame das relações de proximidade geométrica e por projeções em dimensões que podem ser identificadas a partir de pontos no plano e, assim sendo, as categorias mais explicativas das dimensões são as que apresentam maior inércia por dimensão e que, simultaneamente, se situam mais afastadas da origem $(0,0)$.

Para boa interpretação dos resultados, segundo Batista, Escuder e Pereira (2004, p. 630636), deve-se ter em mente que o plano de análise desta técnica tem natureza essencialmente descritiva, não comportando inferências de causa e efeito, e, como corolário, interpretações de risco. O teste $\chi^{2}$ e a análise de resíduos aferem o distanciamento entre as observações realizadas e esperadas por simples aleatoriedade. A análise de correspondência oferece informações de contraste entre relações de categorias de variáveis contingenciadas, de modo que uma relação mais forte entre duas categorias em comparação com outras relações não pressupõe efeitos de uma sobre a outra.

\subsection{Análise de homogeneidade - HOMALS}

A ANACOR, HOMALS e PRINCALS são todas consideradas extensões de técnicas estatísticas clássicas, como a análise de componentes principais para dados nominais. Embora todas essas técnicas tenham em comum a análise de homogeneidade dos dados, tendo como pressuposto a redução dimensional, a HOMALS e a PRINCALS permitem a análise das associações entre dados nominais com mais de duas variáveis, o que não era possível na ANACOR. Assim, sua aplicação mais direta é determinar as associações entre categorias de múltiplas variáveis nominais, além de representá-las em poucas dimensões, normalmente em duas ou três (SPSS, 2000). 
Segundo Meulman e Heiser (2004), esse grupo de técnicas é considerado uma ferramenta adequada para análises exploratórias, tendo como objetivo complementar os modelos obtidos por outras técnicas, reduzindo dimensionalmente os dados a serem analisados.

A HOMALS provê uma representação multivariada da interdependência entre dados não-métricos e busca reduzir as dimensões de estudo de forma a produzir uma solução gráfica, na qual casos com as mesmas categorias de resposta são plotados próximos uns dos outros e casos com diferentes categorias de resposta ficam afastados uns dos outros, em um mapa perceptual (HAIR et al., 2005). Além disso, a HOMALS transforma os dados qualitativos associando-lhes escores que permitam não só a sua representação gráfica, como também a maior separação entre as categorias.

Assim, neste estudo, a HOMALS foi utilizada para identificar as possíveis associações entre quatro variáveis empregadas no estudo para a análise dos impactos dos indicadores de desempenho em cada uma das regiões brasileiras ao longo dos últimos cinco anos.

A HOMALS exige que as observações de cada variável sejam números inteiros positivos e seqüenciais, começando no 1 . Como já mencionado anteriormente, a variável região foi classificada de 1 a 5, e as variáveis dos indicadores foram classificadas de 1 a 3. Além disso, optou-se pela geração de gráficos de apenas duas dimensões, de modo a facilitar a visualização gráfica em um plano cartesiano.

Em suma, o grande objetivo dessa técnica é permitir a análise quantificada da distância entre variáveis, ou seja, à medida que as categorias e os objetos que lhes pertencem estão próximas ou distantes umas das outras.

A seguir, serão apresentados e discutidos os resultados encontrados em função dos métodos acima descritos.

\section{Apresentação dos resultados}

Primeiramente, seguindo as sugestões de Pestana e Gageiro (2000), analisou-se a adequação amostral das variáveis pelo teste qui-quadrado $\left(\chi^{2}\right)$ da ANACOR, para a posterior aplicação da HOMALS. Assim, para cada um dos cinco anos analisados (2002-2006), foram calculados os testes $\chi^{2}$ para a verificação da existência de associação entre a variável região e cada uma das variáveis de desempenho (faturamento por check-out, faturamento por $\mathrm{m}^{2} \mathrm{e}$ faturamento por funcionário). A Tabela 2 apresenta os resultados de cada um dos testes. 
Tabela 2 - Resultados dos testes qui-quadrado $\left(\chi^{2}\right)(2002-2006)$

\begin{tabular}{|c|c|c|c|}
\hline & & Qui-quadrado & Sig. \\
\hline \multirow{3}{*}{2006} & Região x Fat/CO & 39,502 & 0,000 \\
\hline & Região $x$ Fat/m2 & 39,502 & 0,000 \\
\hline & Região x Fat/func & 25,240 & 0,001 \\
\hline \multirow{3}{*}{2005} & Região x Fat/CO & 27,819 & 0,001 \\
\hline & Região x Fat/m2 & 33,257 & 0,000 \\
\hline & Região x Fat/func & 20,078 & 0,010 \\
\hline \multirow{3}{*}{2004} & Região $x$ Fat/CO & 41,280 & 0,000 \\
\hline & Região xFat/m2 & 28,375 & 0,000 \\
\hline & Região x Fat/func & 17,327 & 0,027 \\
\hline \multirow{3}{*}{2003} & Região x Fat/CO & 24,603 & 0,002 \\
\hline & Região $x$ Fat/m2 & 17,327 & 0,027 \\
\hline & Região $x$ Fat/func & 24,036 & 0,002 \\
\hline \multirow{3}{*}{2002} & Região x Fat/CO & 30,284 & 0,000 \\
\hline & Região $\times$ Fat/m2 & 35,053 & 0,000 \\
\hline & Região x Fat/func & 16,815 & 0,032 \\
\hline
\end{tabular}

Por meio da análise da Tabela 2, pode-se observar que todos os testes $\chi^{2}$ foram significantes a um nível de 5\%, o que indica que existe associação entre região e cada uma dos indicadores; assim, é possível a aplicação da HOMALS com as quatro variáveis observacionais do estudo.

\subsection{Análise de homogeneidade}

A partir dos resultados dos testes qui-quadrado, aplicou-se a HOMALS. Foram realizadas as análises com as quatro variáveis (região, faturamento por check-out, faturamento por $\mathrm{m}^{2}$, faturamento por funcionário), para cada um dos anos do estudo (2002-2006), para a verificação do impacto dos três indicadores de desempenho em cada uma das regiões brasileiras ao longo dos últimos cinco anos.

Como primeira verificação dos resultados da HOMALS, são analisadas as medidas de discriminação. Segundo Carvalho (2004), as medidas de discriminação quantificam a variância de cada variável, sendo que quanto mais o seu valor se aproximar do limite superior (i.e., 1), mais as variáveis em questão discriminam os objetos em análise. Além disso, essas medidas mostram as variáveis que mais contribuem para a definição das dimensões. Quanto mais afastada uma variável se encontra do ponto zero, em uma das dimensões, e mais próxima do ponto zero, na outra, mais essa variável é capaz de explicar a primeira dimensão.

A Figura 1 apresenta as medidas de discriminação para cada um dos anos de estudo. 


\begin{tabular}{|c|c|c|c|}
\hline \multicolumn{4}{|c|}{2006} \\
\hline & \multicolumn{2}{|c|}{ Dimensão } & \\
\hline & 1 & 2 & Média \\
\hline Região & 0,070 & 0,151 & 0,110 \\
\hline FaturxCO & 0,929 & 0,943 & 0,936 \\
\hline Faturxm2 & 0,929 & 0,943 & 0,936 \\
\hline Faturxfunc & 0,622 & 0,126 & 0,374 \\
\hline Active Total & 2,550 & 2,162 & 2,356 \\
\hline$\%$ of Variance & 63,8 & 54,1 & 58,9 \\
\hline
\end{tabular}

\begin{tabular}{|c|c|c|c|}
\hline \multicolumn{4}{|c|}{2005} \\
\hline & \multicolumn{2}{|c|}{ Dimensão } & \\
\hline & 1 & 2 & Média \\
\hline Região & 0,094 & 0,154 & 0,124 \\
\hline FaturxCO & 0,831 & 0,566 & 0,698 \\
\hline Faturxm2 & 0,777 & 0,561 & 0,669 \\
\hline Faturxfunc & 0,590 & 0,273 & 0,432 \\
\hline Active Total & 2,292 & 1,553 & 1,922 \\
\hline$\%$ of Variance & 57,3 & 38,8 & 48,1 \\
\hline
\end{tabular}

\begin{tabular}{|c|c|c|c|}
\hline \multicolumn{4}{|c|}{2004} \\
\hline & \multicolumn{2}{|c|}{ Dimensão } & \\
\hline & 1 & 2 & Média \\
\hline Região & 0,128 & 0,097 & 0,113 \\
\hline FaturxCO & 0,836 & 0,665 & 0,751 \\
\hline Faturxm2 & 0,786 & 0,561 & 0,674 \\
\hline Faturxfunc & 0,631 & 0,253 & 0,442 \\
\hline Active Total & 2,382 & 1,577 & 1,979 \\
\hline$\%$ of Variance & 59,5 & 39,4 & 49,5 \\
\hline
\end{tabular}

\begin{tabular}{|c|c|c|c|}
\hline \multicolumn{4}{|c|}{2003} \\
\hline & \multicolumn{2}{|c|}{ Dimensão } & \\
\hline & 1 & 2 & Média \\
\hline Região & 0,206 & 0,043 & 0,125 \\
\hline FaturxCO & 0,802 & 0,604 & 0,703 \\
\hline Faturxm2 & 0,690 & 0,526 & 0,608 \\
\hline Faturxfunc & 0,586 & 0,219 & 0,403 \\
\hline Active Total & 2,285 & 1,392 & 1,838 \\
\hline$\%$ of Variance & 57,1 & 34,8 & 46,0 \\
\hline
\end{tabular}

\begin{tabular}{|c|c|c|c|}
\hline \multicolumn{4}{|c|}{2002} \\
\hline & \multicolumn{2}{|c|}{ Dimensão } & \\
\hline & 1 & 2 & Média \\
\hline Região & 0,240 & 0,031 & 0,136 \\
\hline FaturxCO & 0,810 & 0,660 & 0,735 \\
\hline Faturxm2 & 0,725 & 0,509 & 0,617 \\
\hline Faturxfunc & 0,584 & 0,255 & 0,419 \\
\hline Active Total & 2,359 & 1,456 & 1,908 \\
\hline$\%$ of Variance & 59,0 & 36,4 & 47,7 \\
\hline
\end{tabular}

Figura 1 - Medidas de discriminação (2002 -2006).

A análise da Figura 1 indica que, para todos os anos, as variáveis faturamento por check-out e faturamento por $\mathrm{m}^{2}$ são as que mais discriminam os objetos em análise nas duas dimensões. Assim, os elevados valores obtidos permitem uma desagregação das categorias, discriminando bem cada uma das variáveis, levando, conseqüentemente, à formação de grupos diferenciados de categorias de variáveis (PESTANA e GAGEIRO, 2000). Portanto, desta análise, pode-se inferir que os indicadores que tiveram o maior impacto no comportamento dos grupos supermercadistas brasileiros nos últimos cinco anos foram faturamento por checkout e faturamento por $\mathrm{m}^{2}$.

Os mapas perceptuais derivados da HOMALS são outra forma de interpretação das mudanças ocorridas nas redes supermercadistas nos últimos cinco anos. Segundo Carvalho (2004), quando se dispõe de múltiplos indicadores e sobre os quais se interessa desenvolver uma abordagem que preserve a sua estrutura relacional, a tradução gráfica dos resultados é uma estratégia muito eficaz. Além disso, esse autor cita que a interpretação dos gráficos bidimensionais (mapas perceptuais) é, sem dúvida, o principal objetivo da aplicação da HOMALS quando é necessária a exploração de espaços complexos pela sua multidimensionalidade.

Esse mesmo autor menciona ainda que uma garantia da homogeneidade dos dados é a proximidade registrada entre certas categorias. Assim, a leitura dos planos deve ser realizada entre os quatro quadrantes dos espaços bidimensionais. Dessa forma, a disposição espacial das diferentes configurações em um mesmo espaço será elucidativa das relações de associação ou oposição que os grupos caracterizados por elas estabelecem entre si. 
Assim, para a aplicação da HOMALS, optou-se pela adoção de duas dimensões pelo fato de a interpretação dos resultados ser mais adequada para os propósitos deste estudo. Por meio da normalização pela variável principal, foram elaborados os mapas perceptuais para cada um dos anos em análise (Figuras 2 a 6).

Por meio dos mapas perceptuais apresentados, é possível verificar que existem algumas associações entre as regiões brasileiras e os indicadores de desempenho das redes supermercadistas brasileiras. Primeiramente, verifica-se que para todos os anos do estudo (2002-2006), uma análise dos quatro quadrantes indica a existência de três clusters, onde os tercis concomitantes no tamanho dos três indicadores de desempenho se agrupam com as regiões analisadas.

Assim, no geral, os tercis do tamanho dos três indicadores, por exemplo, fat por co grande, fat por $\mathrm{m}^{2}$ grande e fat por func. grande, apresentam forte associação entre eles, sendo esta uma das razões para a formação dos clusters.

Uma análise ano a ano dos mapas perceptuais indica um comportamento bastante similar entre as redes supermercadistas ao longo de 2006/2005/2004. Nestes três anos, as regiões Sudeste e Norte estão associadas aos indicadores fat por co/fat por $\mathrm{m}^{2} /$ fat por func. grandes, as regiões Sul e Centro-Oeste estão associadas aos indicadores fat por co/fat por $\mathrm{m}^{2} /$ fat por func. grandes e a região Nordeste, aos indicadores fat por co/fat por $\mathrm{m}^{2} /$ fat por func. pequenos.

Esses resultados corroboram com o processo de aquisição de algumas grandes redes supermercadistas pelo WalMart. Esta empresa adquiriu a rede nordestina Bom Preço, com um faturamento de R\$3,4 bilhões, em 2004, e o Sonae, com um faturamento de R\$4,4 bilhões em 2005, sendo que esta rede estava localizada no região Sul. Assim, essas aquisições levaram o WalMart da 6⿳亠 p posição em 2002 e 2003, para a 3a em 2004 e 2005, e, no ano passado, já ocupava a $2^{\underline{a}}$ posição do ranking da SuperHiper por faturamento bruto.

Ademais, a análise do mapa perceptual de 2003 indica exatamente esse processo, pois, neste ano, as regiões Sudeste e Nordeste associam-se aos indicadores fat por co/fat por $\mathrm{m}^{2} /$ fat por func. grandes; as regiões Sul e Centro-Oeste, aos indicadores pequenos; e a região Norte, aos indicadores médios, com uma clara inversão das associações entre as regiões e os tercis dos tamanhos dos indicadores de desempenho.

Por fim, esses resultados indicam uma forma diferenciada da análise do processo de consolidação ocorrido no setor supermercadista brasileiro nos últimos anos. Apesar das técnicas de ANACOR e HOMALS serem exploratórias, a visualização da associação de certas variáveis em um espaço bidimensional é bastante elucidativo e pode fornecer uma indicação do comportamento real do impacto de certas variáveis em outras. 


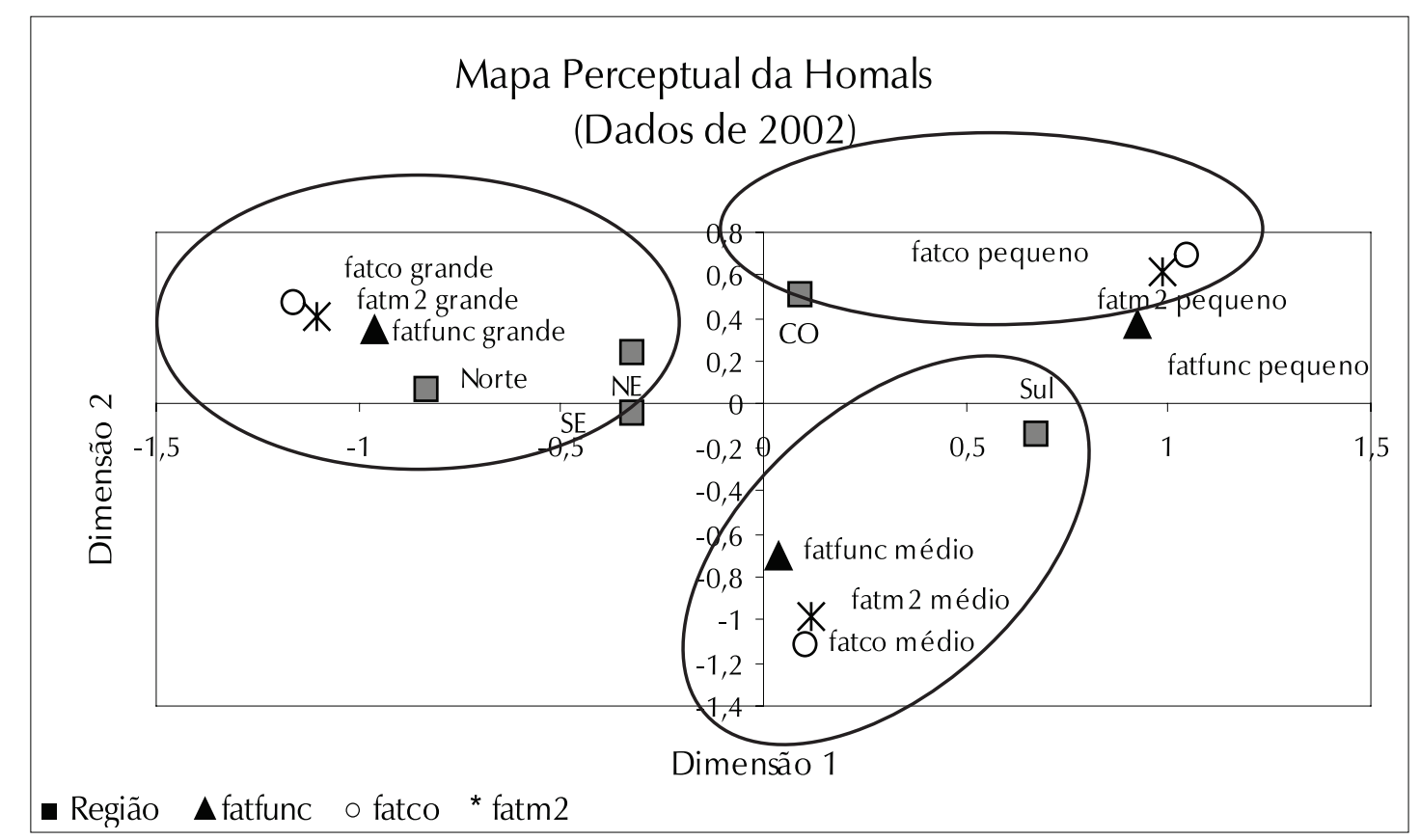

Figura 2 - Mapa perceptual da Homals (2002).

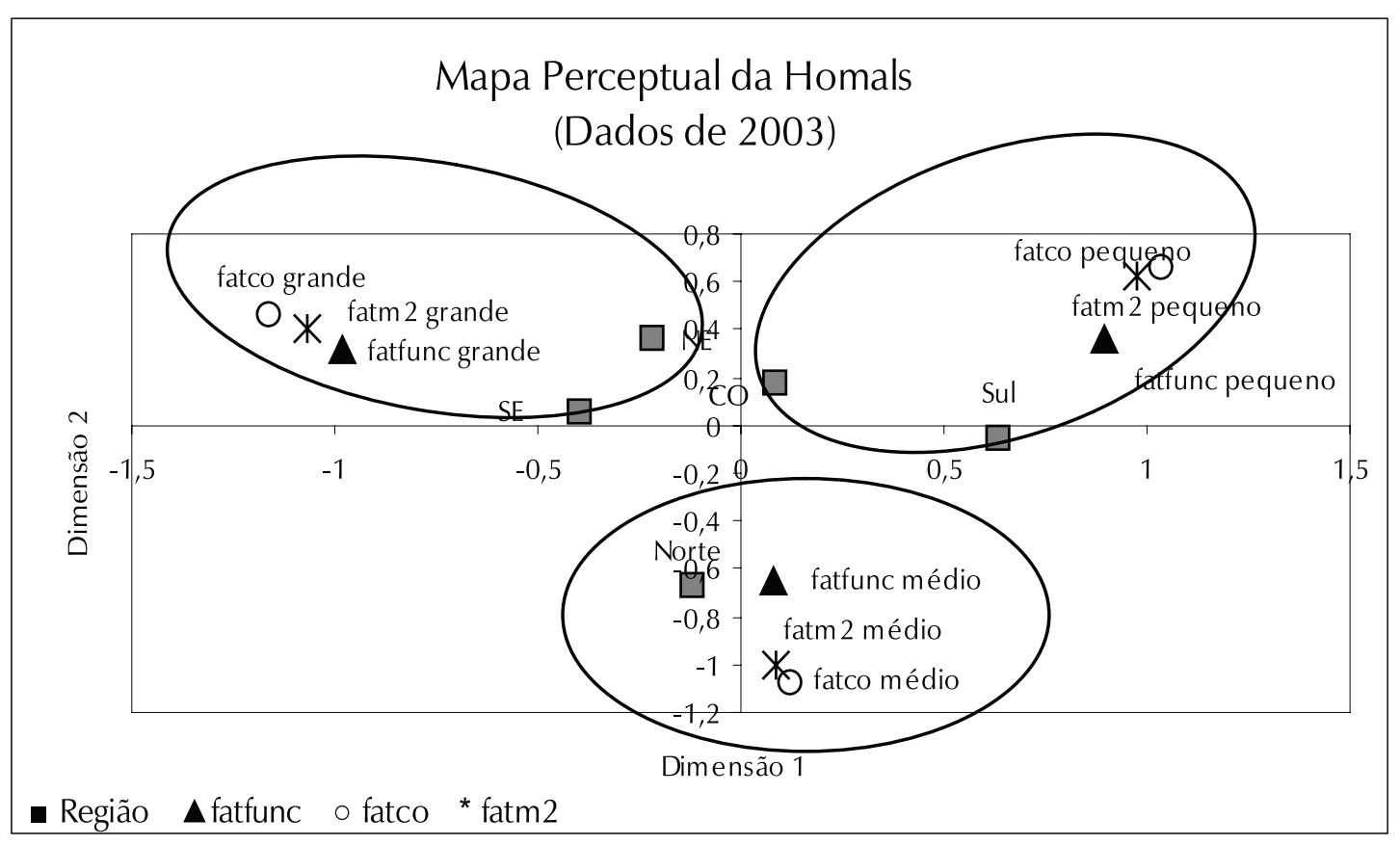

Figura 3 - Mapa perceptual da Homals (2003). 


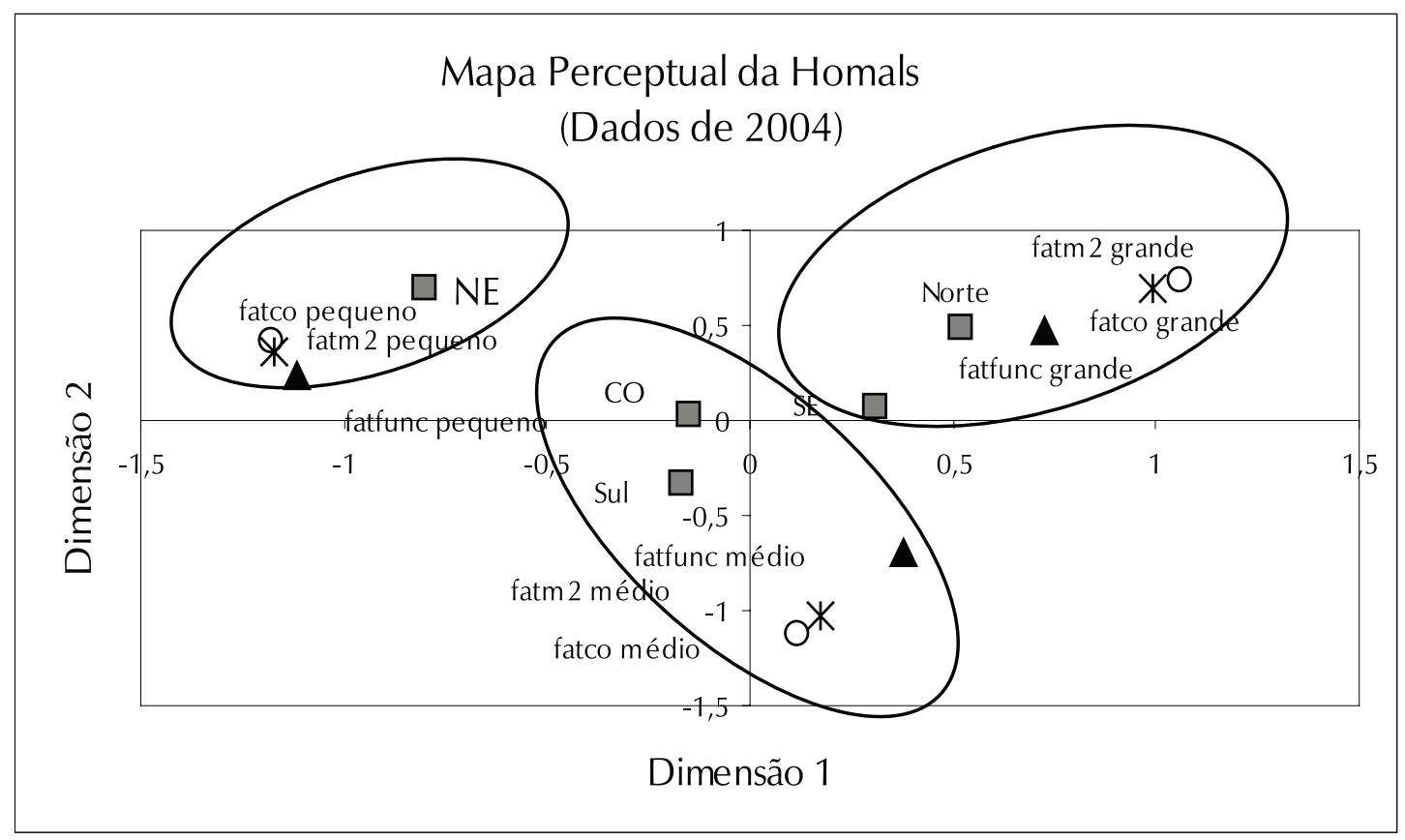

Figura 4 - Mapa perceptual da Homals (2004).

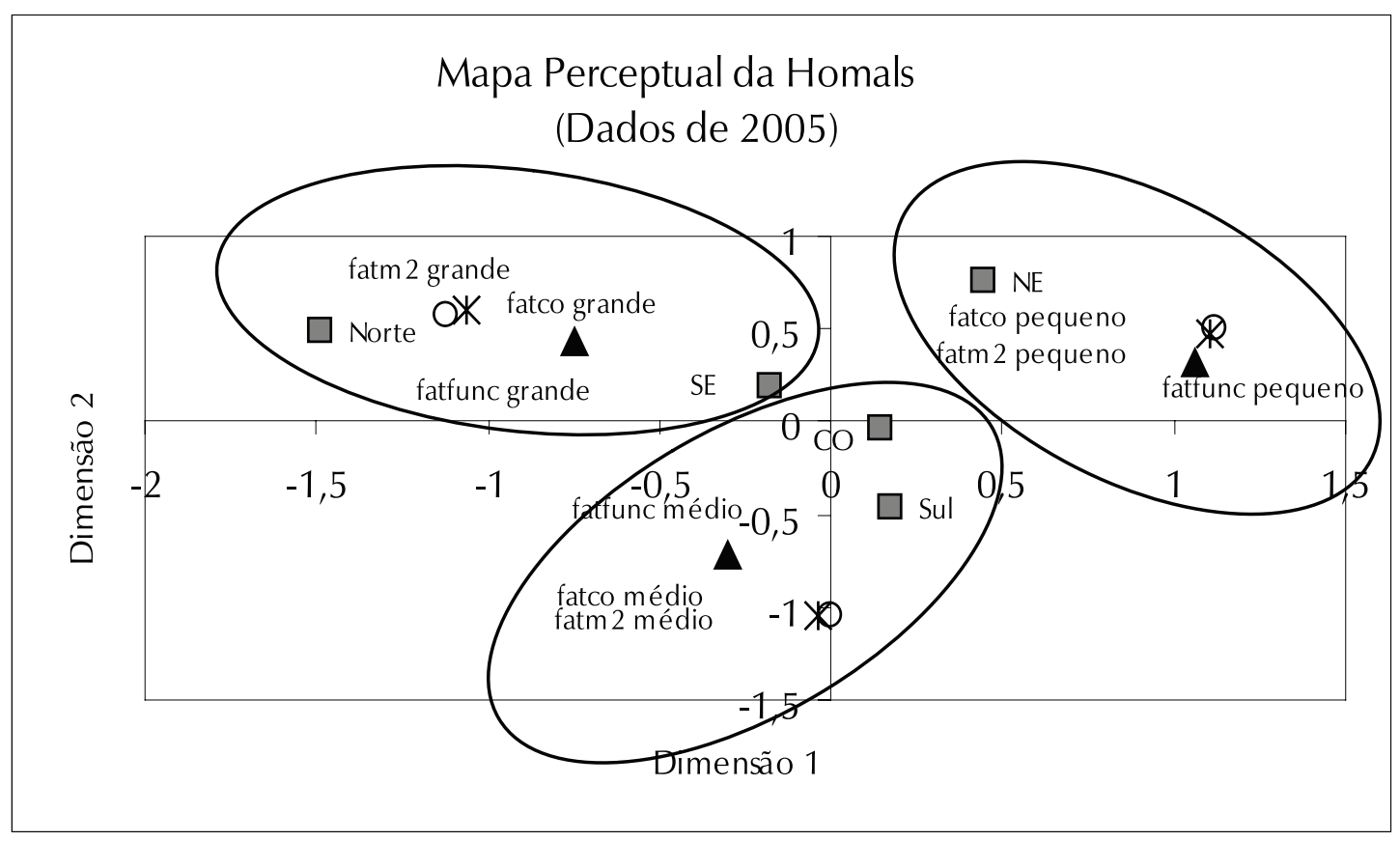

Figura 5 - Mapa perceptual da Homals (2005). 


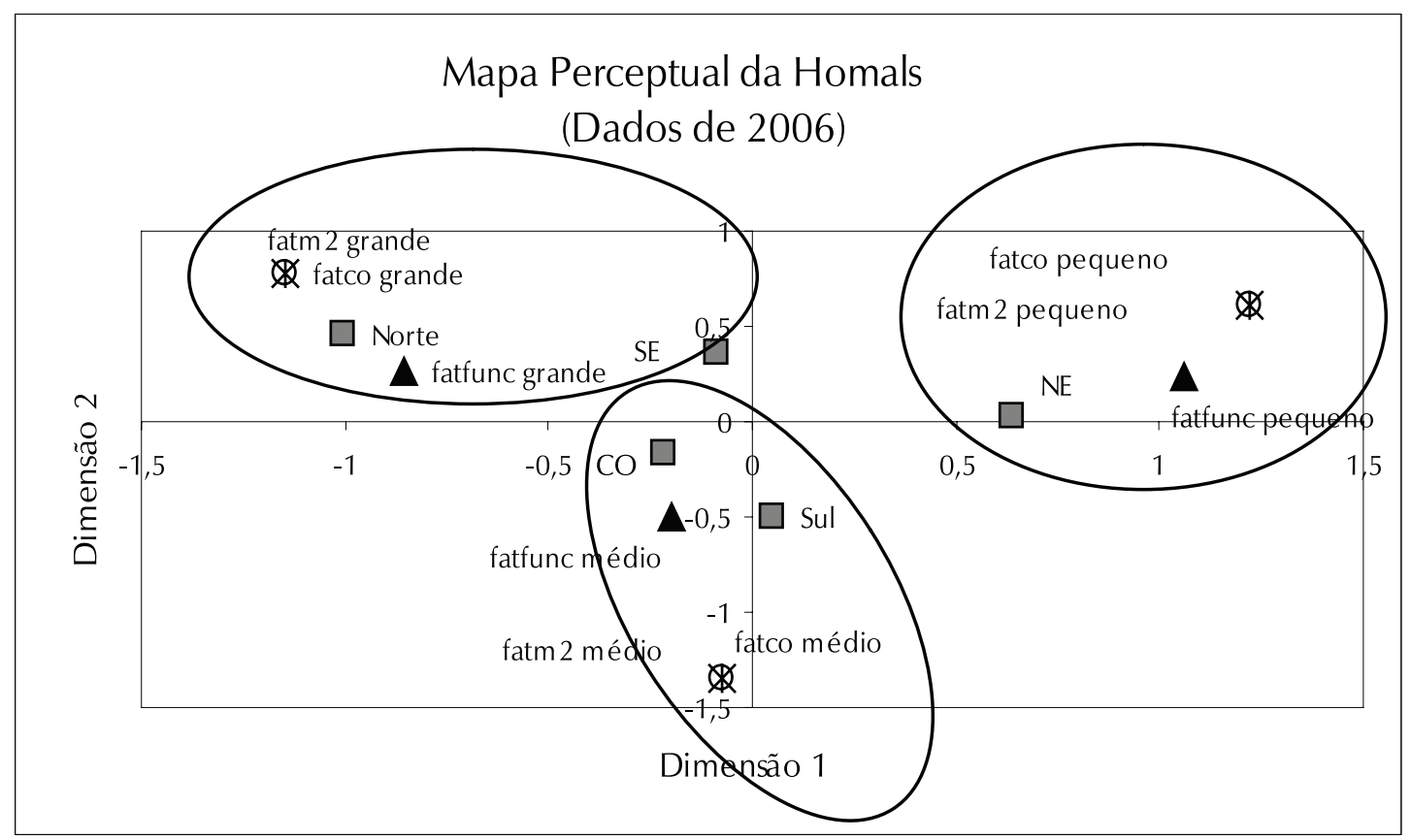

Figura 6 - Mapa perceptual da Homals (2006).

\section{Conclusões e possíveis extensões}

O presente trabalho analisou o comportamento das redes supermercadistas em cada uma das regiões brasileiras, nos últimos cinco anos (2002-2006), em relação a alguns indicadores de desempenho dessas empresas, por meio da aplicação de técnicas de análise de correspondência (ANACOR) e análise de homogeneidade (HOMALS). Os resultados da ANACOR validaram a aplicação da HOMALS com as quatro variáveis observacionais analisadas, e os resultados obtidos indicam os impactos dos processos de aquisição e consolidação ocorridos nesse mercado nos últimos cinco anos.

Primeiramente, é importante ressaltar o poder explicativo das variáveis analisadas sobre o comportamento da atuação de grupos supermercadistas no Brasil. É claro que não se pode ignorar o fato de as técnicas utilizadas neste estudo serem exploratórias e as variáveis representarem um resultado da própria operação organizacional, já que o processo de consolidação propicia um aumento do faturamento e, como conseqüência, um maior aumento total de área de lojas, número de funcionários, expansão de novas lojas e aumento do número de checkouts, porém o incremento destes ao longo do tempo oferece subsídios a esta análise.

Além disso, o aumento da concentração regional e organizacional ao longo dos últimos anos, apresentado neste estudo, demonstra a reestruturação desse mercado, no qual muitas empresas têm expandido as suas participações por meio de aquisições de grupos menores e, dessa forma, a sobrevivência das menores redes passa necessariamente pelo aumento de desempenho diante das gigantes do setor, tanto em gestão de custos, como em termos de vendas e diferenciação (SESSO FILHO, 2001).

Por fim, percebe-se uma nova fase do setor supermercadista no Brasil, com um incremento exacerbado de competitividade, que faz com que os players ofertantes de produtos e serviços apresentem diferenciação no nível de serviços, quer seja pelo gerenciamento de estoques, quer seja pela automação ou pela melhora dos indicadores de atendimento e prazo de entrega. Independentemente de qual seja o principal atributo de diferenciação, é importante 
ressaltar que os aspectos organizacionais analisados neste trabalho representam o sucesso da organização no médio prazo e, portanto, devem ser modelados e avaliados para que sejam determinados padrões e tendências de comportamento.

Dessa forma, o assunto é realmente vasto e instigante. Outros estudos podem relacionar-se com a continuidade da análise, incluindo novas variáveis no modelo, como, por exemplo, o porte das empresas. Além disso, variações dos indicadores em períodos mais longos de tempo podem ser pesquisadas, a fim de se descobrir a sensibilidade de cada fator no comportamento dessas empresas. Outra análise interessante seria a aplicação de técnicas estatísticas multivariadas que comprovassem os resultados deste estudo. Assim, podem ser obtidos resultados mais profundos sobre a atuação varejista no setor de supermercados no Brasil.

\section{Referências}

ANGELO, C. F.; SILVEIRA, J. A. G.; SIQUEIRA, J. P. L. O comportamento de consumo nos supermercados. Pesquisa PROVAR - Programa de Administração de Varejo da FIA, 2001.

BATISTA, L. E.; ESCUDER, M. M. L.; PEREIRA, J. C. R. A cor da morte: causas de óbito segundo características de raça no Estado de São Paulo, 1999 a 2001. Revista de Saúde Pública, v. 38, n. 5, p. 630-636, 2004.

BENZÉCRI, J. P. Correspondence analysis handbook. New York: Marcel Dekker, 1992.

BLECHER, N. Quer preço ou quer marca? Revista Exame, ano 35, n. 12, p. 32, 2001.

CAROLL, J. D.; GREEN, P. E.; SCHAFFER, C. M. Interpoint distance comparisons in correspondence analysis. Journal of Marketing Research, v. 23, n. 3, p. 271280, 1986.

\section{CARVALHO, H. Análise multivariada de} dados qualitativos: utilização da HOMALS com o SPSS. Lisboa: Sílabo, 2004.

CONNOR, J. M.; SCHIEK, W.A. Food processing: an industrial powerhouse in transition. 2. ed. New York: John Wiley \& Sons, 1997.
CYRILLO, D. C. O papel dos supermercados no varejo de alimentos. São Paulo: Instituto de Pesquisas Econômicas, 1987.

DIEESE. O ranking dos supermercados: internacionalização e concentração.

Disponível na URL: <http://www.uol.com. $\mathrm{br} /$ canalexecutivo/notas.htm $>$. Acesso em: 15 out. 2003.

FÁVERO, L. P. L.; BELFIORE, P. P.; FOUTO, N. M. M. D. Escolha de meios de pagamento por populações de média e baixa renda: uma abordagem sob a perspectiva da análise fatorial e de correspondência. Revista de Economia e Administração, v. 5, n. 2, p. 184-200, 2006.

FOX, B. Battle of grocery formats Intensifies: vendors under pressure to choose sides.

Chain Store Age Executive, v.68, n.5, p.48, 1992.

GARRY, M. Dealing with the mass merchants. Progressive Grocer, v.71, n.6, p.73-78, 1992.

. How to stop the beeding.

Progressive Grocer, v.73, n.7, p. 85-88, 1994.

JOHN, B. C. A internacionalização do setor supermercadista paranaense e os 
possíveis efeitos sobre a competitividade do setor. 2000. 48f. Monografia. Faculdade Católica de Administração e Economia, Programa de Iniciação Científica - PAIC, Núcleo de Pesquisa Acadêmica - NPA, Curitiba, 2000.

GREENACRE, M. Theory and applications of correspondence analysis. London: Academic Press, 1984.

GREENACRE, M.; BLASIUS, J.

Correspondence analysis in the social sciences. London: Academic Press, 1994.

GHISI, F. A.; CAMARGO, S. V.; MARTINELLI, D. P. Redes de compras de supermercados de pequeno e médio porte: um estudo multicaso no interior do Estado de São Paulo. Varejo Competitivo, São Paulo, v. 7, p. 217-240, 2003.

HABERMAN, S. J. The analysis of residuals in cross-classified tables. Biometrics, v. 29, n.

1, p. 205-220, 1973.

HAIR, J. F. C. et al. Análise multivariada de dados. 5. ed. Porto Alegre: Bookman, 2005.

HOFFMAN, D. L.; FRANKE, G. R.

Correspondence analysis: graphical representation of categorical data in marketing research. Journal of Marketing

Research, v. 23, n. 3, p. 213-227, 1986.

LUDOVIC, L.; MORINEAU, A.; WARWICK, K. Multivariate descriptive statistical analysis. New York: John Wiley \& Sons Inc., 1984.

MARQUES, E. F.; NORONHA, A.

B. Avaliação e dimensionamento da percepção de excelência de serviços no setor supermercadista: uma abordagem quantitativa. Varejo Competitivo, São Paulo, v. 5, p. 173-197, 2001.

MEULMAN, J.J.; HEISER, W.J. SPSS

Categories 13.0. Chicago: SPSS Inc, 2004.

NISHISATO, S. On quantifying different types of categorical data. Psychometrika, v. 45, n. 4, p. 467-478, 1993.
O GRUPO Pão de Açúcar - Histórico.

Disponível em: <http://www.gpa-ri.com.br/ port/conheca/historico.asp>. Acesso em: 28 abr. 2008.

OLARIAGA, L. J.; HERNÁNDEZ, L. L. Análisis de correspondencias. Madrid: La Muralla, 2000.

O'NEILL, R.E. Close-up clubs. Progressive Grocer, v.71, n.5, p. 61-78, 1992.

ORIGEM e História do Walmart. Disponível em: <http://www.walmartbrasil.com.br/ wmbrazil/wmstores/Mainabout.jsp > . Acesso em: 10 abr. 2008.

PESTANA, M. H.; GAGEIRO, J. N. Análise de dados para ciências sociais: a complementaridade do SPSS. 2. ed. Lisboa: Silabo, 2000.

QUENTAL, C.M.; SILVA, G.F.; LEITE, J. S. Canais de distribuição: associação de pequenos varejistas. Cadernos Discentes Coppead, Rio de Janeiro, n.10, p.5-27, 2001.

ROJO, J. G. Supermercados no Brasil: qualidade, marketing de serviços, comportamento do consumidor. São Paulo: Atlas, 1998.

SESSO FILHO, U. A. Crescimento e desempenho de redes de supermercado na década de 90. Varejo Competitivo, São Paulo, v. 6, p. 21-44, 2001. . O setor supermercadista no Brasil nos anos 1990. 2003. 216f. Tese (Doutorado em Ciências, área de concentração Economia Aplicada) - Escola Superior de Agricultura Luís de Queiroz, Universidade de São Paulo, Piracicaba, 2003.

SHARMA, S. Applied multivariate techniques. New York: John Wiley \& Sons Inc., 1996.

SUPERHIPER. Revista da Associação Brasileira de Supermercados. São Paulo, ano 29, n. 333, maio 2003. 
SUPERHIPER. Revista da Associação

Brasileira de Supermercados. São Paulo, ano 30, n. 345, maio 2004.

SUPERHIPER. Revista da Associação

Brasileira de Supermercados. São Paulo, ano 31, n. 357, maio 2005.

SUPERHIPER. Revista da Associação

Brasileira de Supermercados. São Paulo, ano 32, n. 369, maio 2006.

SUPERHIPER. Revista da Associação

Brasileira de Supermercados. São Paulo, ano 33, n. 381, maio 2007.
TRAJETÓRIA CARREFOUR. Disponível em: $<$ http://www.carrefour.com.br $>$. Acesso em: 10 abr. 2008.

WHITLARK, D. B.; SMITH, S. M.

Using correspondence analysis to map

relationships. Marketing Research, p. 22-

27, outono 2001.

YOUNG, F. Quantitative analysis of

qualitative data. Psychometrika, v. 46, n.4, p. 357-388, 1981. 